\title{
Association of circulating irisin levels with normal weight obesity, glycemic and lipid profile
}

\author{
Sarvenaz Mehrabian ${ }^{1}$, Ehsaneh Taheri ${ }^{2}$, Maryam Karkhaneh ${ }^{1}$, Mostafa Qorbani ${ }^{3,4^{*}}$ and Saeed Hosseini ${ }^{5,1^{*}}$
}

\begin{abstract}
Background: Irisin, a recently identified myokine/adipokine, has potential role in type 2 diabetes and obesity. Normal weight obesity (NWO) is associated with a significantly higher risk of developing metabolic syndrome and cardiometabolic dysfunction. The aim of this study was to investigate association of irisin level with NWO, glycemicand lipid profile in women.
\end{abstract}

Methods: In this matchedcase-control study, 38 subjects with NWO (body mass index (BMI) $<25 \mathrm{~kg} / \mathrm{m} 2$ and $\mathrm{BF} \%>30)$ as case and 26 controls (BMl $<25 \mathrm{~kg} / \mathrm{m} 2$ and $\mathrm{BF} \%<30$ ) were selected randomly from sport clubs in the East area of Tehran, Iran. In addition to anthropometric variables, including BMI and body composition, fasting blood sugar (FBS), fasting levels of irisin andinsulin, triglyceride (TG), total cholesterol (TC), high-density lipoprotein $(\mathrm{HDL})$ and low-density lipoprotein $(\mathrm{LDL})$ cholesterol were measured. All statistical analyses were performed with SPSS 18.0.

Results: In univariate analysis, levels of irisin were significantly higher in subjects with NWO compared to controls ( $0.81 \pm 0.41$ vs. $0.58 \pm 0.26 \mathrm{ng} / \mathrm{ml}, P=0.009)$. This association remained significant after adjusting for confounders (adjusted for energy intake, physical activity, waist circumference and BMI) $(P=0.049)$. In NWO, irisin level was not significantly correlated with all glycemic and lipid profile. In control group, only correlation ofirisin with insulin was statistically significant $(P=0.03)$.

Conclusion: Serum irisin levels were higher in NWO subjects than controls. In control group, only the negative association between irisin and insulin levels was statistically significant.

Further studies with larger sample size are clearly needed to evaluate the potential role of irisin in NWO subject and other disturbed metabolic conditions.

Keywords: Irisin, Normal weight obesity, Glycemic and lipid profile

\section{Background}

Normal weight obesity (NWO) is defined as a condition that is characterized by normal body mass index (BMI: $18.5-24.9 \mathrm{~kg} / \mathrm{m}^{2}$ ) with increased percent body fat. Recent investigations have reported the association of NWO with cardiovascular risk factors, and also with increased

\footnotetext{
* Correspondence: Mqorbani1379@yahoo.com; saeedhmdphd@hotmail.com ${ }^{3}$ Dietary Supplements and Probiotics Research Center, Alborz University of Medical Sciences, Karaj, Iran

${ }^{5}$ Obesity and Eating Habits Research Center, Endocrinology and Metabolism Molecular -Cellular Sciences Institute, Tehran University of Medical Sciences, Tehran, Iran

Full list of author information is available at the end of the article
}

mortality in women [1]. However, the exact prevalence of NWO in the general population is unknown.

The prevalence rates ofhigh waist circumference, waist to hip ratio, high serum levels of low-density lipoprotein (LDL) and triglycerides, low serum level of high-density lipoprotein (HDL), reduce insulin sensitivity (measured as HOMA-IR) and high C-reactive protein levels were higher in patients with NWO compared to subjects with normal weight and normal body fat mass [2].

Increasing evidence suggests that skeletal muscle is an endocrine organ, producing and releasing cytokines which have been termed "myokines" [3, 4]. Several of these myokines are involved in the regulation of physiological 
functions and metabolic pathways, including body metabolism and energy homeostasis $[4,5]$.

Irisin is a newly discovered plasma myokine/adipokine that is produced by the proteolytic cleavage of fibronectin type III domain containing 5 (FNDC5). In skeletal muscle, the peroxisome proliferator-activated receptor $\gamma$ (PPAR- $\gamma$ ) coactivator $1 \alpha($ PGC- $1 \alpha)$ expression as a mediator of the effects of exercise, stimulates increased expression of FNDC5 as a precursor of irisin [6]. In addition to the myocytes, irisin is expressed in adipocytes, kidney, lungand liver tissues. However, the maximum expression of irisin was observed in skeletal muscles [7].

However, conflicting data on the role of irisin or FNDC5, as the precursor of irisin, in obesity and glucose metabolism have recently emerged. Some studies observed the increased blood concentration of irisin and FNDC5 expression in obese patients [8, 9]. In contrast, other studies have shown opposite results $[7,10]$. While some researchers found the negative relationship between bloodirisin level and glycemic parameters [11], others reported that irisin levels positively correlated with the serum concentration of insulin, insulin resistance and fasting blood glucose [12]. In addition, irisin levels were significantly inversely associated with the onset of type 2 diabetes $[13,14]$ and with another pathway, such as inflammation and oxidative stress, which have previously been proposed to be involved in insulin resistance [15].

However, to our knowledge, no study to date has examined the relation between blood irisin levels in patients with the normal weight obesity. Therefore, we aimed to investigate association between irisin levelwithNWO, glycemic and lipid profile in women.

\section{Methods}

\section{Subjects}

This matched case-control study that performed from October 2014 to May 2015, women aged 19 to 39 years were selected randomly from sport clubs in the East area of Tehran, Iran. The study groups included 38 subjects with NWOand 26 healthy subjects as a control group. Two groups were frequency matched based on age groups. Normal weight obesity was defined as those subjects with a normal body mass index (BMI $18.5-24.9 \mathrm{~kg} / \mathrm{m}^{2}$ ) and high body fat percentage (\%BF) content $(\mathrm{BF}>$ $30 \%)$. Subjects with normal BMI $\left(18.5-24.9 \mathrm{~kg} / \mathrm{m}^{2}\right)$ and $\% \mathrm{BF}$ content $<30 \%$ of body weight were considered as controls. The age and BMI were matched between two groups.

Sample size was calculated based on a pilot study and according to two mean comparison formula subject were selected by announcements in the youth sports club in Tehran among them, women aged 19-39 years with normal weight for height who had joined the club recently were selected to measure body composition using the BIA (bio electric impedance analyzer).

Subjects were excluded if they had a history of diabetes, liver and kidney dysfunction, hypertension, gastrointestinal, cardiovascular, thyroid and autoimmune diseases or diagnose infection. Pregnant and lactating individuals were not included andsubjects who used any drugs, supplements or diet for a long time and professional athletes were also excluded from the study.

\section{Ethics statement}

This study was approved by the ethics committee of Endocrinology and Metabolism Clinical Sciences Institute, Tehran University of Medical Sciences (No: 1393-01-981778). All procedures were inaccordance with institutional guidelines and were carried out in compliance with the HelsinkiDeclaration. Before the collection of data, all participants completed written informed consent form.

\section{Measurements of biochemical parameters}

Blood samples were collected after 10-12 h fasting and serum and plasma were separated. All of the samples were stored at $-80^{\circ} \mathrm{C}$ for later biochemical analysis. Fasting serum levels of glucose and lipid profiles, including total cholesterol (TC), triglyceride (TG) and high density lipoprotein (HDL-C) were measured by a Hitachi 912autoanalyser (Hitachi, Mannheim, Germany) using commercial kits. Low density lipoprotein (LDL) was calculated by friedewald equation. Insulin was measured by radioimmunoassay. Serum irisin concentration was measured using the enzyme-linked immunosorbent assay (ELISA) kits (Biovendor Laboratory Medicine, Modrice, Czech Republic).

\section{Body composition measurements}

After removal shoes and heavy clothing, weight and height were measured using a Seca scale (Seca725 GmbH \& Co. Hamburg, Germany), to the nearest $0.1 \mathrm{~cm}$ and $0.1 \mathrm{~kg}$, respectively for each participant. BMIwas calculated as weight (in $\mathrm{kg}$ ) the square of height in meters. Waist circumference was measured by a plastic tape measure locating above the uppermost lateral border of the right ilium, at the end of a normal expiration, to the nearest $0.1 \mathrm{~cm}$ in a horizontal plane. Hip circumference was measured by placing the tape around the hips at the biggest circumference of the buttocks using non-stretchable tape measures. Body composition, including total body fat mass and fatfree mass were assessed using Tanitabody composition analyzer (Model TBF-300; Tanita, Tokyo, Japan) during fasting state and before blood sampling. Subjects were required to remove all metal objects, such as earrings, etc., and to wear light clothing before each measurement. The device calculates body fat percentage, fat 
mass, and fat free mass and predicts muscle mass on the basis of data using BIA.

\section{Statistical analysis}

The normal distribution assumption of continuous variables was assessed using Shapiro-wilk test. Data was expressed as mean $\pm \mathrm{SD}$. The analysis ofcovariance (ANCOVA) test was used tocompare mean of irisin level between NWO and controls after adjustment for confounders in threedifferent models. In model I crude association was assessed. In model II energy intake, physical activity, waist circumference (WC) was adjustedand in model III additionally BMI was adjusted. Spearman correlation coefficients were used to determine the relationship between irisin levels and glycemic and lipid profile. All statistical analyses were performed using SPSS version 18 (SPSS Inc., Chicago, IL). The level of significance was considered as $\leq 0.05$.

\section{Results}

Clinical and laboratory findings and demographic characteristics of the study groups are presented in Table 1 . The mean age of subjects were participated in this study was $28.95 \pm 4.63$ years, which was not significantly different between NWO and control groups.

Table 1 Baseline anthropometric, clinical and laboratory characteristics of normal weight obese (NWO) subjects and healthy controls

\begin{tabular}{lccc}
\hline Variables & NWO $(n=38)$ & Controls $(n=26)$ & $P$-value \\
\hline Age (year) & $28.76 \pm 4.67$ & $29.23 \pm 4.50$ & 0.69 \\
Height $(\mathrm{cm})$ & $165.89 \pm 4.43$ & $165.32 \pm 4.81$ & 0.62 \\
Weight $(\mathrm{kg})$ & $62.77 \pm 4.77$ & $56.98 \pm 4.40$ & $<0.0001$ \\
BMI $\left(\mathrm{kg} / \mathrm{m}^{2}\right)$ & $22.26 \pm 1.23$ & $20.88 \pm 1.28$ & $<0.0001$ \\
Waist circumference $(\mathrm{cm})$ & $74.77 \pm 4.74$ & $70.84 \pm 3.03$ & $<0.0001$ \\
Hip circumference $(\mathrm{cm})$ & $98.90 \pm 4.29$ & $93.44 \pm 2.99$ & $<0.0001$ \\
WHR & $0.75 \pm 0.04$ & $0.77 \pm 0.03$ & 0.66 \\
Body fat $(\%)$ & $32.66 \pm 2.47$ & $23.79 \pm 1.62$ & $<0.0001$ \\
Body fat $(\mathrm{kg})$ & $20.47 \pm 2.71$ & $13.56 \pm 1.45$ & $<0.0001$ \\
DBP $(\mathrm{mmHg})$ & $70.66 \pm 13.6$ & $75.50 \pm 12.9$ & 0.57 \\
SBP $(\mathrm{mmHg})$ & $90.76 \pm 12.6$ & $91.5 \pm 17.1$ & 0.11 \\
Total cholesterol $(\mathrm{mg} / \mathrm{dl})$ & $174.00 \pm 29.35$ & $174.1 \pm 21.44$ & 0.97 \\
LDL- cholesterol $(\mathrm{mg} / \mathrm{dl})$ & $90.89 \pm 18.08$ & $89.53 \pm 17.07$ & 0.76 \\
HDL- cholesterol $(\mathrm{mg} / \mathrm{dl})$ & $59.02 \pm 13.70$ & $61.11 \pm 9.00$ & 0.46 \\
TG (mg/dl) & $87.07 \pm 28.28$ & $82.92 \pm 26.67$ & 0.55 \\
FBS (mg/dl) & $87.71 \pm 8.16$ & $84.5 \pm 7.3$ & $<0.0001$ \\
Insulin $(\mu l \mathrm{l} / \mathrm{ml})$ & $9.02 \pm 4.75$ & $6.31 \pm 2.49$ & $<0.0001$ \\
\hline Data are expred & & &
\end{tabular}

Data are expressed as mean \pm SD $P<0.05$ is statistically significant

NWO normal weight obesity, $B M I$ body mass index, WHR waist to hip ratio, $D B P$ diastolic blood pressure, $S B P$ systolic blood pressure, $L D L$ low density lipoprotein, $H D L$ high-density lipoprotein, $T G$ triglyceride
The mean \pm SD for weight, waist and hip circumferences and $\mathrm{BF} \%$ were higher significantly in subjects with NWO compared to controls.

The results showed that thelevelof LDL, TG, FBS and insulin in subjects with NWO were higher than control subjects which was statistically significant for fasting levels of insulin and glucose.

Table 2 shows the crude and adjusted mean levels of irisin in NWO and control groups. In crude model circulating irisin levels were significantly higher in subjects with NWO compared with controls $(0.81 \pm 0.41 \mathrm{vs}$ $0.58 \pm 0.26 \mathrm{ng} / \mathrm{ml}, P=0.009)$. This association remained significant after adjusting for potential confounders in model II $(P=0.02)$ and III $(P=0.049)$.

The correlation between circulating irisin levels and biochemical variables are shown in Table 3. In NWO, irisin level was not significantly correlated with all glycemic and lipid profile. In control group, only correlation of irisin with insulin was statistically significant $(P=0.03)$.

\section{Discussion}

The current findings indicated the significantly higher circulating levelof irisinin NWO subjects compared to controls who had normal weight and normal BF\%. However, previous trials reported the increased blood level of irisin in the obese subjects. In this context, Saleh et al.found significantly increased irisin level in obese andoverweight women compared to normal weight ones [16].

Similarly, the reports of Ivanov et al. [17], Stengel et al. [10], and Wen et al.[18] showed the positive association between irisin levels and BMI in healthy nondiabetic subjects. The results of Stengle et al. [10] and Huh et al. [7] studies indicated that circulating levels of irisin were higher in healthy subjects with morbid obesity than normal weight controls. On the other hand, Liu et al. reported that circulating irisin level had a negative association with BMI, waist to hip ratio and $\mathrm{BF} \%$ in men [13]. However in this study, body composition was not measured directly.

It has been suggested that $\mathrm{BF} \%$ is a better indicator oftotal adiposity compared to BMI. In support of this statement, our results showed that in NWO subject who

Table 2 Serum level of irisin in subjects with normal weight obesity (NWO) compared to controls

\begin{tabular}{lccc}
\hline Circulating irisin level $(\mathrm{ng} / \mathrm{ml})$ & NWO $(n=38)$ & Controls $(n=26)$ & $P$-value \\
\hline Model 1 & $0.81 \pm 0.41$ & $0.58 \pm 0.26$ & 0.009 \\
Model 2 & $0.81 \pm 0.36$ & $0.59 \pm 0.35$ & 0.02 \\
Model 3 & $0.82 \pm 0.36$ & $0.58 \pm 0.40$ & 0.049 \\
\hline
\end{tabular}

Model 1: No adjustment

Model 2: adjusted for energy intake, physical activity, waist circumference Model 3: adjusted for energy intake, physical activity, waist circumference and BMI

${ }^{*} P$-value is for ANCOVA test, $P<0.05$ is statistically significant 
Table 3 Spearman's correlation coefficients between serum level of irisin and glycemia/ lipid profile in normal weight obese (NWO) subjects and controls

\begin{tabular}{|c|c|c|c|c|}
\hline \multirow[t]{2}{*}{ Variables } & \multicolumn{2}{|c|}{ NWO $(n=38)$} & \multicolumn{2}{|c|}{ Controls $(n=26)$} \\
\hline & $r$ & $P$-value* & $r$ & $P$-value* \\
\hline Total cholesterol (mg/dl) & -0.02 & 0.89 & 0.28 & 0.15 \\
\hline LDL- cholesterol (mg/dl) & 0.001 & 0.99 & -0.23 & 0.25 \\
\hline HDL- cholesterol (mg/dl) & 0.06 & 0.68 & 0.05 & 0.78 \\
\hline $\mathrm{TG}(\mathrm{mg} / \mathrm{dl})$ & 0.11 & 0.48 & -0.06 & 0.75 \\
\hline $\mathrm{FBS}(\mathrm{mg} / \mathrm{dl})$ & 0.06 & 0.69 & -0.08 & 0.74 \\
\hline Insulin ( $\mu \mid \cup / m l)$ & 0.13 & 0.4 & -0.42 & 0.03 \\
\hline
\end{tabular}

* $P$-value is for spearman correlation, $P<0.05$ is statistically significant

had normal range of $\mathrm{BMI}$ and higher $\mathrm{BF} \%$, the serum level of irisin was significantly higher than controls.

Regarding to possible mechanism, it is suggested that increased circulating irisin in obesity is an adaptive compensatory response to obesity-induced disturbed metabolism such as decreased insulin level [16]. Alternatively," irisin resistance" may be another description for increased levels of irisin in obesity, as has already established for leptin or insulin in obesity [11].

According to our observation in this study, serum irisin levelcorrelated positively with FBS and insulin levels in NWO subjects. This correlation was negative in controls, although it was only significant between irisin and insulin level in controls.

Gomer et al. reported positive correlation between irisin level and HbA1c in T2D patients with and without obesity [19]. Liu et al. showed the positive association between circulating irisin and FBS in non-obese, non-diabetic individuals [13]. In consistence to this results, Huh et al., Stengel et al. and Liu et al. showed positive correlation between serum levels of irisin and FBS $[7,10,13]$. Data from our study showed that the serum levels of FBS and insulin were higher significantly in NWO patients compared to controls. Another study speculated that long time exposure to high blood glucose, irrespective of BMI, was associated significantly with decreased serum level of irisin in diabetic patients $[7,13]$. Contrary to type 2 diabetes and despite to higher level of FBS and insulin, NWO patients had higher levels of irisin. Patients with NWO are susceptible to development of T2D; therefore, it is possible that the irisin levels could be decreased in long-time in NWO subjects. In addition, the high serum level of irisin in NWO subjects might be compensatory response to condition called "irisin resistance", similar to T2 DM.

In agreement with this result, our study showed that the adipose tissue might be the main source of irisin secretion in NWO subjects, because NWO subjects had a higher $\mathrm{BF} \%$ than controls.

Previous studies found that the activity oftranscriptional co-activator PPAR- $\gamma$ co-activator-1 $\alpha(\mathrm{PGC} 1 \alpha)$, a molecule up-stream of irisin, in skeletal muscles and therefore circulating irisin level was lower in patients with type 2 diabetes or pre-diabetes than healthy obese subjects [20-22].

On the other hand, in patients with abnormal blood glucose or T2DM, the expressions of FNDC5/irisin are decreased in adipose tissue and skeletal muscles.

Saleh et al. suggested that the glucose intolerance may gradually up regulate the skeletal muscles expression of FNDC5/irisin in non-diabetic subjects [23]. According to this statement and despite to the results of previous studies, we expect the high circulating irisin level in patients with T2DM who are exposed to high level of glucose. To illustrate this conflict observation, we mentioned the results of other studies suggested timelyregulation of local and circulating irisin with tissue-specific mechanisms in different physiological status such as obesity, pre-diabetes and T2DM [24, 25]. Also, Choi et al. and Huh et al. showed that decreased blood irisin level could expose subjects to the development of insulin resistance and T2DM $[7,14]$. Similar to the results of Saleh et al., study, we found that serum irisin was significantly negatively associated with insulin level in control subjects [16].

Interestingly, it was shown the opposite expression of FNDC5 in skeletal muscle and adipose tissue of T2D patients compared to lean, obese and pre-diabetic subjects [26]. Diabetic patients displayed the lowest expression of FNDC5 in adipose tissue and circulating irisin level. The muscle expression of FNDC5 was similar in diabetic patients and obese subjects. It is suggested that glucose and lipid, not insulin might be inhibitory regulatory factor in muscle FNDC5 expression in metabolic disorders. It is plausible to think that different interaction exist between expressions of FNDC5 in skeletal muscle and adipose tissue in patients with NWO who had increased insulin and glucose levels. In addition, this expression might change during long term dependant to the glycemic control in NWO subjects.

Our current data also revealed a positive correlation between irisin and HDL-c, LDL-c, TG and a negative correlation association between irisin and TC in NWO subjects. In addition, our results indicate that circulating irisin correlated positively with HDL-c and TCand correlated negatively with LDL-c and TG in control group.

A limited study was performed to evaluate the association between irisin and lipid profile in various populations with obesity or diabetes and both of them. Liu et al. reported that serum level of irisin correlated positively with total cholesterol and TG in non- obese, non- diabetic subjects [13]. The significant association between irisin and HDL-c in healthy non-diabetic subjects in Benton et al. study, suggested a protective role for circulating irisin in cardiovascular disease [27], which is similar to our result in NWO and controls. Another study conducted by 
Sanchis-Gomer et al., reveals no significant relationship between irisin and TC, TG and LDL [28]. In contrary, Wen et al. study showed a significantpositive correlations of TC, TG and LDL with circulating irisin in non- diabetic participants [18]. The positive association between serum level of irisin and TG was shown in Liu et al. study [13]. Previous studies showed that muscle FNDC5 was positively associated with TG [7], and negatively associated with HDL-c [7, 12]. For the first time, Saleh et al. reported that serum irisin has a positive relationship with both leptin and insulin in type 2 diabetes [16]. It is plausible to think that the association between irisin and lipid profile may be mediated by its effect on leptin.

The correlation of irisin with TG, LDL-c and HDL-c was inversely between NWO and control subjects. More studies are needed to establish the detail association between irisin and lipid profiles in subjects with NWO compared to obese, diabetes and non- obese, non-diabetic healthy subjects. In addition to skeletal muscles and adipose tissue, FNDC5 mRNA/irisin detected in various tissues, including pericardium, kidney, liver, lung and neurons in human [7, 9, 29-31]. It was suggested thatthe muscle/adipose irisin secretion ratio might vary dependent to the physiological status. In athletes with trained muscles by exercise, muscle tissue would actively increase FNDC5/irisin but, in obesity, the adipose tissue is the main source of FNDC5/ irisin secretion [32].

The limitations of our study are its small sample size and lack of information about the FNDC5 expression and irisin level in adipose tissue, skeletal muscles and its ratio in subjects with normal weight obesity. There was no data about the life quality and socioeconomic factors in our study that considered as a limitation of our study. The strength of our study is that this is the first study that investigated blood irisin level and its association with biochemical parameters in subjects with normal weight obesity.

\section{Conclusion}

In summary, the results of this study demonstratedserum irisin levels were higher in NWO subjects than controls. In control group, only the negative association between irisin and insulin levels was statistically significant. Further studies with larger sample size are clearly needed to evaluate the potential role of irisin in NWO subject and other disturbed metabolic conditions.

\footnotetext{
Abbreviation

BAT, brown adipose tissue; BIA, bio electrical impedance analysis; BMI, body mass index; FBS, fasting blood sugar; FFM, free fat mass; FM, fat mass; FNDC5, fibronectin type 111 domain 5; HDL, high density lipoprotein; LDL, low density lipoprotein; NOW, normal weight obesity; PGC1 a, PPARYcoactivator-1a; REE, resting energy expenditure; UCP, uncoupling proteins; WAT, wight adipose tissue; WHR, waist to hip ratio
}

\section{Acknowledgements}

We would like to thank all the patients who kindly participated in the study. This research project has been supported by a grant from Tehran University of Medical Sciences (TUMS).

\section{Funding}

This study was supported by a grant from the EMRI from TUMSwith the projects number, 1393-01-98-1778.

\section{Availability of data and materials}

The datasets supporting the conclusions of this article are included within the article.

\section{Authors' contributions}

SM and MK participated in the gathering of the data. SM and ET drafted the manuscript. MQ participated in the design of the study and performed the statistical analysis. SH and ET designed and supervised the study and

reviewed the manuscript. All authors read and approved the final manuscript.

\section{Competing interests}

The authors declare that they have no competing interests.

\section{Statement of consent for publication}

Formed consent for publication of clinical data wassigned by the patient.

\section{Ethics approval and consent to participate}

This study was approved by the ethics committee of Endocrinology and Metabolism Research Institute (EMRI), Tehran University of Medical Sciences (TUMS) with the projects number, 1393-01-98-1778.

\section{Author details}

'Department of Clinical Nutrition, School of Nutritional Sciences and Dietetic, Tehran University of Medical Sciences, No 44, Hojjat-dost Alley, Naderi St., Keshavarz Blvd, Tehran 1416-643931, Iran. ${ }^{2}$ Endocrinology and Metabolism Research Center, Endocrinology and Metabolism Research Institute, Tehran University of Medical Sciences, Tehran, Iran. ${ }^{3}$ Dietary Supplements and Probiotics Research Center, Alborz University of Medical Sciences, Karaj, Iran. ${ }^{4}$ Chronic Diseases Research Center, Endocrinology and Metabolism Population Sciences Institute, Tehran University of Medical Sciences, Tehran, Iran. ${ }^{5}$ Obesity and Eating Habits Research Center, Endocrinology and Metabolism Molecular-Cellular Sciences Institute, Tehran University of Medical Sciences, Tehran, Iran.

Received: 15 March 2016 Accepted: 14 June 2016 Published online: 27 June 2016

\section{References}

1. Romero-Corral A, Somers VK, Sierra-Johnson J, Korenfeld Y, Boarin S, Korinek J, et al. Normal weight obesity, a risk factor for cardiometabolic dysregulation and cardiovascular mortality. Eur Heart J. 2010;31(6):737-46.

2. Litwin SE. Normal weight obesity is bigger really badder? Circ Cardiovasc Imaging. 2012;5(3),286-8.

3. Febbraio MA, Pedersen BK. Contraction-induced myokine production and release, is skeletal muscle an endocrine organ? Exerc Sport Sci Rev. 2005;33(3),114-9.

4. Pedersen BK, Febbraio MA. Muscles, exercise and obesity, skeletal muscle as a secretory organ. Nat Rev Endocrionol. 2012;8(8),457-65.

5. Pardo M, Roca-Rivada A, Seoane LM, Casanueva FF. Obesidomics, contribution of adipose tissue secretome analysis to obesity research. Endocrine. 2012:41(3),374-83.

6. Boström P, Wu J, Jedrychowski MP, Korde A, Ye L, Lo JC, et al. A PGC1-[agr]dependent myokine that drives brown-fat-like development of white fat and thermogenesis. Nature. 2012;481(7382),463-8.

7. Huh JY, Panagiotou G, Mougios V, Brinkoetter M, Vamvini MT, Schneider BE, et al. FNDC5 and irisin in humans, I. Predictors of circulating concentrations in serum and plasma and II. mRNA expression and circulating concentrations in response to weight loss and exercise. Metabolism. 2012;61(12),1725-38.

8. Gutierrez-Repiso C, Garcia-Serrano S, Rodriguez-Pacheco F, Garcia-Escobar E, Haro-Mora JJ, Garcia-Arnes J, et al. FNDC5 could be regulated by leptin in adipose tissue. Eur J Clin Invest. 2014;44(10),918-25.

9. Moreno-Navarrete JM, Ortega F, Serrano M, Guerra E, Pardo G, Tinahones F, et al. Irisin is expressed and produced by human muscle and adipose tissue 
in association with obesity and insulin resistance. J Clin Endocrinol Metab. 2013;98(4),E769-78.

10. Stengel A, Hofmann T, Goebel-Stengel M, Elbelt U, Kobelt P, Klapp BF. Circulating levels of irisin in patients with anorexia nervosa and different stages of obesity-correlation with body mass index. Peptides. 2013;39,125-30.

11. Lopez-Legarrea P, de la Iglesia R, Crujeiras AB, Pardo M, Casanueva FF, Zulet $\mathrm{M}$, et al. Higher baseline irisin concentrations are associated with greater reductions in glycemia and insulinemia after weight loss in obese subjects. Nutr Diabetes. 2014;4(2),e110.

12. Hee Park K, Zaichenko L, Brinkoetter M, Thakkar B, Sahin-Efe A, Joung KE, et al. Circulating irisin in relation to insulin resistance and the metabolic syndrome. J Clin Endocrinol Metab. 2013;98(12),4899-907.

13. Liu J-J, Wong MD, Toy WC, Tan CS, Liu S, Ng XW, et al. Lower circulating irisin is associated with type 2 diabetes mellitus. J Diabetes Complications. 2013:27(4),365-9.

14. Choi Y-K, Kim M-K, Bae KH, Seo H-A, Jeong J-Y, Lee W-K, et al. Serum irisin levels in new-onset type 2 diabetes. Diabetes Res Clin Pract. 2013;100(1),96-101.

15. Gratas-Delamarche A, Derbré F, Vincent S, Cillard J. Physical inactivity, insulin resistance, and the oxidative-inflammatory loop. Free Radic Res. 2014;48(1),93-108.

16. Saleh $\mathrm{O}$, Majeed M, Oreaby G. Descriptive consideration of serum irisin levels various factors, obesity, type 2 diabetes mellitus, pre-diabetic status, gender, and athletics. J Diabetes Metab. 2014;5(471),2.

17. Ivanov IP, Firth AE, Michel AM, Atkins JF, Baranov PV. Identification of evolutionarily conserved non-AUG-initiated N-terminal extensions in human coding sequences. Nucleic Acids Res. 2011;39(10),4220-34.

18. Wen M-S, Wang C-Y, Lin S-L, Hung K-C. Decrease in irisin in patients with chronic kidney disease. 2013.

19. Sanchis-Gomar F, Lippi G, Mayero S, Perez-Quilis C, García-Giménez JL. Irisin, a new potential hormonal target for the treatment of obesity and type 2 diabetes. J Diabetes. 2012;4(3),196.

20. Benton CR, Nickerson JG, Lally J, Han XX, Holloway GP, Glatz JF, et al. Modest PGC-1alpha overexpression in muscle in vivo is sufficient to increase insulin sensitivity and palmitate oxidation in subsarcolemmal, not intermyofibrillar, mitochondria. J Biol Chem. 2008;283(7),4228-40. Epub 2007/12/15.

21. Gillies CL, Abrams KR, Lambert PC, Cooper NJ, Sutton AJ, Hsu RT, et al, Pharmacological and lifestyle interventions to prevent or delay type 2 diabetes in people with impaired glucose tolerance, systematic review and meta-analysis. BMJ. 2007;334(7588),299.

22. Kelly DP. Irisin, light my fire. Science. 2012;336(6077),42-3.

23. Saleh $\mathrm{O}$, Majeed MJ, Oreaby GM. Descriptive consideration of serum irisin levels various factors, obesity, type 2 diabetes mellitus, pre-diabetic status, gender, and athletics. J Diabetes Metab. 2014;5:471.

24. Kurdiova T, Balaz M, Vician M, Maderova D, Vlcek M, Valkovic L, et al. Effects of obesity, diabetes and exercise on Fndc5 gene expression and irisin release in human skeletal muscle and adipose tissue, in vivo and in vitro studies. J Physiol. 2014;592(5),1091-107.

25. Pekkala S, Wiklund PK, Hulmi JJ, Ahtiainen JP, Horttanainen M, Pöllänen E, et al. Are skeletal muscle FNDC5 gene expression and irisin release regulated by exercise and related to health? J Physiol. 2013;591 (21),5393-400.

26. Koksharova EO, Mayorov AY, Shestakova MV, Dedov II. Metabolic characteristics and therapeutic potential of brown and 'beige' adipose tissues. Diabetes Mellitus. 2014;2014(4),5-15.

27. Benton C, Holloway G, Han X-X, Yoshida Y, Snook L, Lally J, et al. Increased levels of peroxisome proliferator-activated receptor gamma, coactivator 1 alpha (PGC-1a) improve lipid utilisation, insulin signalling and glucose transport in skeletal muscle of lean and insulin-resistant obese Zucker rats. Diabetologia. 2010;53(9),2008-19.

28. Sanchis-Gomar F, Alis R, Pareja-Galeano H, Sola E, Victor VM, Rocha M, et al. Circulating irisin levels are not correlated with BMl, age, and other biological parameters in obese and diabetic patients. Endocrine. 2014;46(3),674-7.

29. Bordicchia M, Liu D, Amri E-Z, Ailhaud G, Dessi-Fulgheri P, Zhang C, et al. Cardiac natriuretic peptides act via p38 MAPK to induce the brown fat thermogenic program in mouse and human adipocytes. J Clin Invest. 2012;122(3),1022.

30. Dun SL, Lyu R-M, Chen Y-H, Chang J-K, Luo JJ, Dun NJ. Irisin-immunoreactivity in neural and non-neural cells of the rodent. Neuroscience. 2013;240,155-62.

31. Roca-Rivada A, Castelao C, Senin LL, Landrove MO, Baltar J, Crujeiras AB, et al. FNDC5/irisin is not only a myokine but also an adipokine. PLoS One. 2013;8(4),e60563.

32. Timmons JA, Baar K, Davidsen PK, Atherton PJ. Is irisin a human exercise gene? Nature. 2012;488(7413),E9-10.

\section{Submit your next manuscript to BioMed Central and we will help you at every step:}

- We accept pre-submission inquiries

- Our selector tool helps you to find the most relevant journal

- We provide round the clock customer support

- Convenient online submission

- Thorough peer review

- Inclusion in PubMed and all major indexing services

- Maximum visibility for your research

Submit your manuscript at www.biomedcentral.com/submit 